\title{
Editorial
}

\section{A revision in the definition of osteoporosis}

\author{
George P. Lyritis, Stavroula Rizou \\ Hellenic Osteoporosis Foundation, Athens, Greece
}

All published work is licensed under Creative Common License CC BY-NC-SA 4.O (Attribution-NonCommercial-ShareAlike)

The term 'osteoporosis' first appeared in 1833 as a description of a pathological state of the bone. French pathologist, Jean Martin Lobstein ${ }^{1,2}$ coined this term using a combination of two Greek words, ostoun (bone) and poros (hole) to describe the condition as one having holes in the bone associated with fragility. While this term was successful and descriptive of the osseous atrophy and mechanical insufficiency the word 'osteoporosis' was incorporated in the medical vocabulary only in the twentieth century by the famous American Endocrinologist Fuller Albright (1941). This was mainly as a result of accelerated postmenopausal bone loss, increased incidence of vertebral fractures and other fractures of the appendicular skeleton ${ }^{3}$. It is, therefore, clear that since then the fate of osteoporosis was parallel with the clinical increasing interest about menopause and the important role of estrogen insufficiency ${ }^{4}$.

\section{The history of the definition of osteoporosis}

From time to time, the term "osteoporosis" has been widely used by researchers and clinicians in the most significant laboratory and clinical findings. These were used in order to differentiate osteoporotic patients from normal cases or from those with similar diseases, additionally, for monitoring the consequences of the disease and especially unjustified fractures.

In 1941, Fuller Albright defined osteoporosis as: "too little formation of calcified bone" 5 , a definition which only encompasses the quantitative aspect of the disease and not the qualitative. In 1988, the American National Institute of Health (NIH) published its first definition, in which osteoporosis is referred to as "a condition in which the bone mass diminishes, increasing susceptibility of bones to suffer fractures" 6 . Osteoporosis is a disorder characterized by low bone density, microarchitectural deterioration of bone tissue and a consequent increase in fracture risk ${ }^{7}$. Since "low bone density" was not clearly defined previously, in 1994 the World Health Organization (WHO) defined osteoporosis as a bone density measured with DXA, 2.5 standard deviations (SD) below the mean for healthy women aged 20-29 years, also referred to as a T-score of $-2.5 \mathrm{SD}$. The definition of osteoporosis as defined by the WHO in 1994 remains unchanged. the $\mathrm{NIH}$ in the year 2001, updating the earlier definition of 1988, which it considered "a disease of the whole skeleton characterized by a low bone mass and an alteration in the bone microarchitecture which causes fragile bone, the consequence of which is an increased risk of fractures" 8 .

From the above it is obvious that the osteoporosis definition tries to describe the clinical and laboratory findings of the disease while at the same time aims to differentiate osteoporosis from other relative diseases and to define the threshold values of bone loss beyond which clinical manifestations are present. Research on the etiology of bone frailty continues at a rapid pace constantly indicating new laboratory or clinical parameters which redefine osteoporosis.

\section{Definition of osteoporosis on the basis of bone density}

The use of bone densitometry on patients with possible osteoporosis gives the opportunity to modify the osteoporosis definition from a more qualitative indication of bone tissue to one with specific quantitative parameters. The osteoporosis definition by the World Health Organization stipulates osteoporosis as an excess of a statistical deviation of the surface bone density value, measured through X-ray absorptiometry and through the evaluation of the population on values below -2.5 SD from the mean value (Figure 1).

Patients with bone density below -2.5 SD were considered to have an increased possibility of osteoporotic fractures not only to axial skeleton but also to extremities. The classification of osteoporotic and normal cases creates an extensive undefined category of patients with bone density above -2.5 SD but below - 1 SD. These patients with a BMD T-score between - 1 and -2.5 SD are described as osteopenic; also considered as having an increased risk for an osteoporotic fracture occurrence. There was a controversy concerning the way of evaluation of bone loss; whether this should be compared to people with normal bone density of the same age and gender or alternatively compare the difference between the existent bone density and the

Corresponding author: George P. Lyritis, 5 Ag. Varvaras str. 14562 Kifissia, Athens, Greece

E-mail: glyritis@heliost.gr

Accepted 26 March 2016 


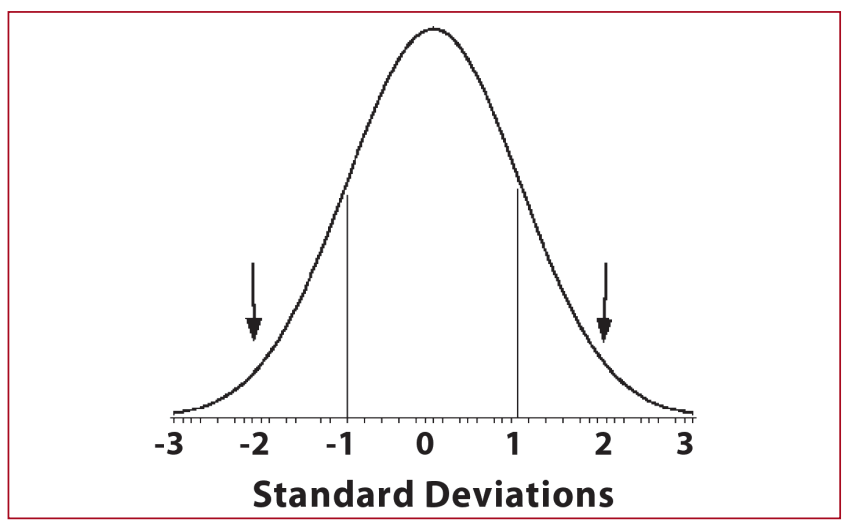

Figure 1. This diagram represents the normal distribution of bone density.

peak bone density of the specific gender. The osteoporosis definition based on T-score cut-off point of -2.5 SD prevailed not only for diagnosis but also as an indication for antiosteoporotic treatment.

Definitively determining of the $-2.5 \mathrm{SD}$ as a threshold was subject to debate arising from epidemiological studies in which it is shown that the majority of osteoporotic fractures happen to individuals with BMD T-scores in the osteopenic range. Although fracture risk increases with a decrease in BMD, the vast majority of osteoporotic fractures occur in osteopenic patients ${ }^{9}$. This is due to the fact that even though the risk of fracture is lower in osteopenia than in osteoporosis, the number of subjects at risk is much higher in the osteopenic range due to the Gaussian distribution of BMD values in the population. However despite the controversy, bone density remains one of the most valid and reliable measure of fracture risk assessment and determining pharmacological treatment for patients with prevalent osteoporotic fractures.

\section{Proposal for a more simplified definition of osteoporosis}

Main aims of the diagnostic and therapeutic approach of osteoporosis should be to provide: 1) facilitation of clinicians to identify specific causes that lead to increased fracture risk and 2) guidance for individualized osteoporosis treatment relevant to each patient.

A more simplified osteoporosis definition which complies with the above logic is the following: Osteoporosis is an increased risk of an occurrence of unjustified fractures accompanied by internal bone problems and external factors.

\section{Internal problems explained}

Internal problems are considered disorders of bone texture. The latter is defined as alterations of the external and internal geometry of the bone, impaired phases of bone remodeling, as well as the inability of bone tissue to repair micro-damages or the fact that is partially repairs them. This inability stems from continuous cumulative mechanical loading or the problems caused by accelerated bone loss. Similar factors that could possibly be considered internal bone structure problems are numerous, therefore they will not discussed further. One can reasonably infer that most of the internal bone problems on osteoporotic patients are not easy to detect using the currently available diagnostic methods, such as bone densitometry or biochemical markers, available to clinicians at present. Most cases of osteoporotic patients' frailty syndrome-which is possibly caused by internal bone problems- have to be treated with antiresorptive or osteoanabolic agents; known to effectively reduce the risk of osteoporotic fractures based on long-term prospective studies ${ }^{10-18}$.

The weakness of implementating a more personalized pharmaceutical treatment on osteoporotic patients with internal bone problems is obvious. Although, the antiosteoporotic drugs should have different efficacy depending on the different types of internal bone problems, this is hindered by the lack of suitable diagnostic methods thus failing to distinguishing the exact nature of these problems.

\section{External causes of unjustified fractures explained}

External causes, which lead to osteoporotic fractures, are mainly considered falls which are responsible for the osteoporotic fractures on the peripheral skeleton.

There are a number of causes leading to instability and falls which in turn lead to movement instability and balance disorders. The above mentioned causes are related to the appearance of specific comorbidities such as disorders of heart rhythm, neurological issues, disorders of vision, deafness, psychological-mental conditions and musculoskeletal disorders (osteomalacic myopathy, sarcopenia, osteoarthritis etc).

\section{Variability of fracture risk}

The possibility of an osteoporotic fracture to occur is significantly increased if the reason that caused it exceeds a fracture threshold. This limit is well established in the osteoporosis definition based on bone densitometry and it refers to T-score <-2.5 SD. As previously reported, this threshold is not applicable since the majority of fractures occur in the osteopenic range. Presently clinicians do not have at their disposal an undisputed osteoporosis threshold concerning laboratory tests, something that is hopefully to be expected imminently. Since, the importance of fracture risk is commonly accepted; all implemented therapeutic methods should retain individuals patients' fracture risk below that threshold.

The fracture risk might flactuate even on the same patient due to the continuous interactions of the internal and external reasons which have been reported. This aspect 
should find implementation on therapeutic decisions for osteoporotic patients. For example a woman 70 years old with an osteoporotic vertebral fracture, which occurred 25 years ago, without any fracture occurring since then is speculated to have lower fracture risk than a woman of the same age, same laboratory findings and having an osteoporotic fracture at exactly the same vertebra but having occurred more recently. The alteration of fracture risk should be accompanied by reconsideration of therapeutic targets. It is essential to perform long-term prospective studies to investigate the importance of variability of fracture threshold.

In conclusion, the treating physician should choose the most suitable treatment for the patient based on medical history, fracture risk, previous treatment for osteoporosis, as well as future follow-up treatments ${ }^{19}$.

\section{References}

1. Lobstein JF. Traité d'anatomie Pathologique. Paris: Chez F.G. Levrault; 1833.

2. Lobstein JF. Von der Knochenbrüchigkeit oder Osteopsathyose. ed. Lehrbuch der pathologischen Anatomie. Stuttgart: Thieme; 1835:179.

3. Albright F, Smith P, Richardson A. Postemenopausal osteoporosis. Trans Assoc Am Physicians 1940; 55:298-305.

4. Bijlsma AY, Meskers CG, Westendorp RG, Maier AB. Chronology of age-related disease definitions: osteoporosis and sarcopenia. Ageing Res Rev 2012; 11 (2):320-4

5. Albright F, Reifenstein EC. The parathyroid glands and metabolic bone disease; selected studies. Baltimore, Williams \& Wilkins 1948.

6. Osteoporosis. National Institutes of Health Consensus Development Conference Statement. Natl Inst Health Consens Dev Conf Consens Statement 1984; 5:p6.

7. Consensus Development Conference Diagnosis, prophylaxis and treatment of osteoporosis Am J Med 1993; 94:646-650.

8. Assessment of fracture risk and its application to screening for postmenopausal osteoporosis. Report of a WHO Study Group. World Health Organ Tech Rep Ser 1994; 843:1-129.

9. Siris ES, Miller PD, Barrett-Connor E, et al. Identification and fracture outcomes of undiagnosed low bone mineral density in postmenopausal women: results from the National Osteoporosis Risk Assessment. JAMA 200 1; 286(22):2815-22.

10. Black DM, Cummings SR, Karpf DB, et al. Randomised trial of effect of alendronate on risk of fracture in women with existing vertebral fractures. Fracture Intervention Trial Research Group. Lancet 1996; 348(9041): 1535-41.

11. Black DM, Thompson DE, Bauer DC, et al. Fracture risk reduction with alendronate in women with osteoporosis: the Fracture Intervention Trial. FIT Research Group. J Clin Endocrinol Metab 2000; 85(11):4118-24.

12. Felsenberg D, Alenfeld F, Bock O, et al. Placebo-controlled multicenter study of oral alendronate in postmenopausal osteoporotic women. FOSIT-Study-Group. Fosamax International Trial. Maturitas 1998; 31(1):35-44.

13. Reginster J, Minne HW, Sorensen $\mathrm{OH}$, et al. Randomized trial of the effects of risedronate on vertebral fractures in women with established postmenopausal osteoporosis. Vertebral Efficacy with Risedronate Therapy (VERT) Study Group. Osteoporos Int 2000; 11(1):83-91.

14. Adami S, Libanati C, Boonen S, et al. Denosumab treatment in postmenopausal women with osteoporosis does not interfere with fracture-healing: results from the FREEDOM trial. J Bone Joint Surg Am 2012; 94(23):2113-9.

15. Papapoulos S, Lippuner K, Roux C, et al. The effect of 8 or 5 years of denosumab treatment in postmenopausal women with osteoporosis: results from the FREEDOM Extension study. Osteoporos Int 2015; 26(12):2773-83.

16. Adami S. Protelos: nonvertebral and hip antifracture efficacy in postmenopausal osteoporosis. Bone 2006; 38(2 Suppl 1):23-7.

17. Meunier PJ, Slosman DO, Delmas PD, et al. Strontium ranelate: dose-dependent effects in established postmenopausal vertebral osteoporosis - a 2-year randomized placebo controlled trial. J Clin Endocrinol Metab 2002; 87(5):2060-6.

18. Body JJ, Gaich GA, Scheele WH, et al. A randomized double-blind trial to compare the efficacy of teriparatide [recombinant human parathyroid hormone (1-34)] with alendronate in postmenopausal women with osteoporosis. J Clin Endocrinol Metab 2002; 87(10):4528-35.

19. Makras, P, G. Vaiopoulos, G. P. Lyritis. 2011 guidelines for the diagnosis and treatment of osteoporosis in Greece. J Musculoskelet Neuronal Interact 2012; 12(1):38-42. 